\title{
Population genetic structure of endemic fish species facilitating their survival in changing environments - a case study on the genus Telestes in Croatia
}

\author{
Ivana Buj ${ }^{1}$, Zoran Marčić ${ }^{2}$, Elena Flauder ${ }^{2}$, Marko Ćaleta ${ }^{3}$, Radek Šanda ${ }^{4}$, and Jasna \\ Vukić ${ }^{5}$ \\ ${ }^{1}$ University of Zagreb \\ ${ }^{2}$ University of Zagreb Faculty of Science \\ ${ }^{3}$ University of Zagreb Faculty of Teacher Education \\ ${ }^{4}$ National museum \\ ${ }^{5}$ Charles University
}

June 23, 2020

\begin{abstract}
he genus Telestes comprises primarily freshwater fishes distributed mostly in the Mediterranean area. Recent investigation of the evolutionary history of this genus revealed that it originated in southern Europe, where the most ancient Telestes species are still present. Isolation of rivers in the karstic region facilitated allopatric speciation resulting in a high number of Telestes species and a great portion of endemics in freshwaters of Croatia and Bosnia and Herzegovina. Most of the endemic Telestes species have very small distribution areas, inhabiting a single river or few water bodies in a single karstic field, making them extremely vulnerable to all anthropogenic changes. In order to contribute to practical conservation of the endemic Telestes species through design of conservational measures that are likely to be the most effective in ensuring future viability and undisturbed evolutionary course of those species, we have investigated their population genetic structure and estimated their viabilities. Population viability analyses were carried out based on the current state of populations and their habitats, as well as recognized threats. Several scenarios included also potential threats. Our results show that invasive species pose the most dangerous threats to the future survival of the endemic Telestes species. Contrary to previous opinions that a reduced genetic diversity is characteristic for small populations, high intraspecific genetic diversity was revealed inside most of the investigated species, which might enable most of the populations to cope with future changes and mitigate negative effects.
\end{abstract}

\section{Introduction}

Endemic species, particularly if distributed on a very small area, are posing a tough set of problems in conservation biology and their protection is complex and challenging for several reasons (see Frankham, 1995). Since they are distributed in a small, restricted area, each threat occurring there is usually affecting the whole species or a great portion of its individuals. Moreover, there is no or only limited possibility for population augmentation or reintroduction. And finally, it is often considered that such range restricted and small population size species, distributed in unique areas, have small genetic diversity (lowered by genetic drift, Frankham, 1996, 1998; Furlan et al., 2012) and low effective population size, reducing their evolutionary potential and increasing extinction risk (Markert et al., 2010). In small populations, the importance of genetic drift relative to selection is increased and beneficial alleles are usually thought to be lost. On the other hand, deleterious alleles are more likely to reach high frequencies in comparison with the situation in larger populations (Whitlock, 2000). Thereafter, small populations are usually considered to have lowered 
fitness and are more prone to extinction. The reduced genetic diversity, often thought to be characteristic for small populations, is making them less able to respond to environmental changes than larger populations (Whitlock, 2000).

The genus Telestes Bonaparte, 1837 (family Leuciscidae, order Cypriniformes, class Actinopterygii) comprises primarily freshwater fishes distributed mostly in the Mediterranean area. Ecological conditions in their habitats include moderately cold, flowing or stagnant, well oxygenated, clean water (Marčić, 2013). Recent investigation of the evolutionary history of the genus (Buj et al., 2017) revealed that the origin of Telestes genus occurred in southern Europe, where the most ancient Telestes species are still present. Moreover, especially high diversity of Telestes at species and intraspecific levels was revealed in the Adriatic watershed in Croatia and Bosnia and Herzegovina, as a consequence of a complex geological history of the region leading to triple colonization of that area by distinct Telestes lineages. Isolation of rivers in this karstic area facilitated allopatric speciation resulting in high number of Telestes species and great portion of endemics in freshwaters of Croatia and Bosnia and Herzegovina. Out of 14 currently recognized Telestes species, as much as ten are distributed in the mentioned area (Buj et al., 2017; Caleta et al., 2019), nine of which are endemics, with very restricted distribution ranges. Telestes croaticus (Steindachner, 1866) inhabits only four small karstic rivers (Jadova, Suvaja, Ričica and Obsenica), located in the Lika karstic region in Croatia; T. tursky (Heckel, 1843) has distribution range restricted to a small Čikola River, tributary of the Krka River; while T. ukliva (Heckel, 1843) is restricted to the Cetina River basin, also in Croatia. Telestes miloradi Bogutskaya, Zupančič, Bogut \& Naseka, 2012 is a recently described species found only in small springs and streams in the Konavosko karstic field in Croatia. Recent investigation (Buj et al., 2017) disputed distinctiveness of T. dabar Bogutskaya, Zupančič, Bogut \& Naseka, 2012, described from the Dabar karstic field in Bosnia and Herzegovina. Nevertheless, T. metohiensis (Steindachner, 1901) is distributed in the watersheds of the karstic fields in southern Bosnia and Herzegovina. All the mentioned species are endemic species inhabiting small distribution areas in the Adriatic watershed. Three more endemic species have extremely small distribution ranges located at the border line between the Adriatic and the Black Sea watersheds in Croatia, but belonging to the Black Sea watershed due to the underground connections. Those three species are: T. fontinalis (Karaman, 1972) from the Krbavsko karstic field, T. polylepis (Steindachner, 1866) known from a single locality - Šmit lake, and T. karsticus Marčić \& Mrakovčić, 2011, inhabiting few small streams in mountain region in Croatia.

Exceptionally high diversity of Telestes in freshwaters of middle and southern Croatia and Bosnia and Herzegovina is a result of several phenomena:

- Origin of the genus most probably took place in the mentioned region and colonization started from there (Buj et al., 2017)

- Complex geological history led to triple colonization by genetically distinct Telestes lineages (Buj et al., 2017)

- Isolation of karstic rivers and lakes is promoting allopatric speciation (e.g. Buj et al., 2015; Buj et al., 2020)

- Undisturbed evolutionary development of populations in rivers that were not affected by glaciations, resulting in their high genetic diversities (e.g. Buj et al., 2015).

Most of the endemic Telestes species have very small distribution areas, inhabiting a single river or few watersheds in a single karstic field (Ćaleta et al., 2015; Buj et al., 2017), making them extremely vulnerable to all anthropogenic changes affecting their limited habitats. Furthermore, it is possible that their effective population sizes are low, due to small habitat size, but also due to particular environmental conditions, which include dry seasons, lowering of the water level and droughts of part or the whole water stream. There are indications that some, if not all Telestes species, migrate in underground shelters during dry season (personal observation) and maybe even use underground water channels for migrations, similarly as proved for the genus Delminichthys (Palandačić et al., 2012) that also inhabits karstic watershed in the same area. Unfortunately, habitats and populations of these endemic species were not spared from significant anthropogenic threats and on some localities, there are plans for even greater impact, regardless 
of invaluable importance of these unique biodiversity components. Previous investigation already revealed that, out of six endemic Telestes species further investigated in this research, only T. karsticus turned out to have lower intraspecific genetic diversity, whereas the remaining five species comprised high levels of genetic diversity (Buj et al., 2017). In this investigation, we analyzed intrapopulational genetic diversities, as well as relationships among populations in species comprising more than a single population. Furthermore, in order to contribute to practical conservation of the endemic Telestes species, particularly to design of conservational measures that are likely to be the most effective in ensuring future viability and undisturbed evolutionary course of those species, we have investigated their population genetic structure and estimated their viabilities. Population viability analyses were carried out based on the current status of populations and habitats, as well as currently recognized and potential threats.

\section{Material and methods}

This investigation comprised population genetic and viability analyses of six Telestes species distributed in northern and central Dinaric karst in Croatia, for which we were able to obtain satisfactory sample for population genetic analyses. We have obtained samples from altogether 12 localities. Fishes were caught by electrofishing and small part of fin tissues from each individual was preserved in ethanol until further analyses. Fin clips were taken after fish were anesthetized. Laboratory protocols for DNA isolation and polymerase-chain reactions (PCR) were described in Buj et al. (2017), since part of samples used in that analysis, was further investigated in this research. In order to obtain reliable results of the population genetic analyses, additional samples were included in this investigation (Table 1).

In order to describe intraspecific structure of the endemic Telestes species that comprise more than one population (T. karsticus, T. croaticus, T. ukliva), we have calculated genetic differentiation between populations of the same species, as well as estimated gene flow between them. Level of genetic differentiation among populations was estimated using DnaSP v.5. software (Librado \& Rozas, 2009). We have calculated statistics based on haplotypes $\left(\chi^{2}\right.$ test and $\left.H \mathrm{ST}\right)$ and the ones based on nucleotide sequences $\left(K \mathrm{ST}, K{ }_{\mathrm{ST}}{ }^{*}, K\right.$ $\mathrm{s}^{*}, Z$ and $\left.Z^{*}\right)$. The null hypothesis that there is no genetic diversity between two populations was rejected based on the permutation test, if $\mathrm{p}<0.05$. Interactions among populations were further investigated using maximum likelihood approach (Beerli, 1998; Beerli \& Felsenstein, 2001) implemented in MIGRATE 3.2.1 (Beerli, 2009). We estimated immigration rates as mutation-scaled effective immigration rates (M), which measures the importance of immigrants vs. mutation when bringing new variants into the population, and also as the number of immigrants per generation $(\mathrm{Nm})$.

Future viability of six endemic species was analysed using Population Viability Analysis (PVA), implemented in the Vortex Version 10.2.6.0 software (Lacey \& Pollak, 2017). Vortex uses simulation model to carry population or populations through life cycle, predicting and quantifying survival (as well as extinction rates), based on the data implemented in the model in order to describe population and habitat, as well as present and/or predicted threats and/or conservational measures. We have estimated extinction risk of populations and species in the next 100 years. Number of repetitions for each scenario was 1000 . We have designed several scenarios for each species, one of which (scenario 0) did not comprise any catastrophes, yet it was based on the observed status of populations and their habitats. Five additional scenarios comprised modifications of environmental factors in habitats of Telestes populations, as well as invasive species effects. These scenarios are based on the realistic presumptions on how habitat characters might be deteriorated in the future, as well as expectations about answers of Telestes populations based on literature data and personal observations. As already stated, Scenario 0 is based on the current status of populations, without additional threats. For the input data for effective population sizes and migrations among populations, results of this investigation were used and maximum likelihood estimates for both parameters for all investigated populations were included. Input data on the reproductive system, sex ratio, reproductive rates and mortality rates were based on previous investigations and literature data (Marčić, 2013; Vuković, 1985; Zanella, 2003; Zanella et al., 2009). For description of ecological characters of Telestes species, we have used available data on this genus. Namely, all investigated Telestes species are small species, living in similar karstic environments, occupying similar ecological niche and with similar requirements regarding habitat conditions. Thereafter, 
we have described reproductive system of all investigated Telestes species as polyginous with age of the first offspring for both females and males being 1+. Maximum lifespan was estimated to be six years and we have included possibility of reproduction until maximum age. Number of broods per year for the investigated Telestes species is one and the average number of progeny per brood that was included in PVA simulation is 1500 (based on investigation of Marčić, 2013). Sex ratio by birth is considered to be 1:1 in normal conditions. The only exceptions regarding the sex ratios were Scenarios 3 and 4 , as will be explained later. Scenario 1 presumes water quality changes (due to water pollution, oxygen level lowering or any other anthropogenic impact), so that habitat conditions become suboptimal for Telestes species, expressed by lowering of reproductive rates for $20 \%$ and increase of mortality rates of the offspring for $5 \%$. Scenario 2 predicts the realistic possibility of invasive species spreading and their negative impact on Telestes populations. Negative effects of invasive species are presumed to lower reproductive rates for $10 \%$ and increase mortality rates for $10 \%$ for the youngest age classes and $7 \%$ for older ages. A Scenario 3 , forecasts water temperature raising due to climate changes and its effect on Telestes populations by means of skewed sex ratio at birth in favor of males (predicts $70 \%$ of males by birth). Such phenomenon of skewed ratio has already been estimated in some Telestes populations (Marčić, 2013; Krivokapić, 1992) and its connection with elevated water temperature levels suggested (Marčić, 2013). In several investigations (Marčić, 2013; Krivokapić, 1992) various sex ratios diverging from 1:1 were reported, between $61 \%$ and $77 \%$ of males at birth, so in the Scenario 3 we have included the average value, leaving all other parameters unchanged (the same as in the Scenario 0). Scenario 4 encompasses simultaneous effects of the water quality deterioration, invasive species and temperature raising due to climate changes. Unfortunately, simultaneous action of various threats have been widely observed, leading to the extinction vortex of populations, so this scenario is also realistic and possible. Negative effects of all three threats acting at the same time was described as skewed sex ratio (70\% of males by birth), reduced reproductive rates (by 20\%) due to deterioration of habitat conditions and increased mortality rates (for $15 \%$ in the youngest age classes and $7 \%$ in $2+$ and further classes) as a consequence of effects of both, invasive species and suboptimal habitat conditions. Scenario 5, on the other hand, does not predict continuous lowering of any of the habitat conditions, yet it encompasses catastrophic events (defined in Vortex as events that significantly reduce survival and/or reproduction potential of population). Catastrophes included in the Scenario 5 were predicted to occur twice in 100 years, destroying $50 \%$ of populations and completely disabling reproduction in the year of occurrence. Catastrophes included can be of different types (e.g. extreme drought events as a consequence of climate changes, extreme pollutions, habitat modifications, diseases etc.), because we did not specify their type, yet we specified their effects on the endemic Telestes populations.

It is important to notice that, obviously, it is not possible to have a full and complete forecast of the future events and we cannot know for sure what kind of threats will affect which population nor when. It is, furthermore, possible that effects of the predicted threats will be more or less severe than predicted. Nevertheless, we have prepared realistic PVA scenarios, acting in a same way on various Telestes species and populations, which is an approach that shall enable us pinpointing the most dangerous threats for each species, comparison of answers of various Telestes species and populations to the same threat, as well as quantifying and modelling their extinction risk in the next 100 years. Such information are very valuable for conservation purposes.

\section{Results}

Previous investigation (Buj et al., 2017) already revealed high genetic diversities of the majority of Telestes species distributed in the karstic watersheds in Croatia, explained by long term evolutionary history in favorable conditions and the lack of bottlenecks during longer geologic periods. This investigation revealed intrapopulational diversities and differences between them. Intrapopulational diversities of all populations comprised under T. croaticus and T. ukliva are very high (Table 2). Populations of T. karsticuscontain the lowest levels of genetic diversities, with all samples from Studenac expressing the same haplotype (no genetic diversity whatsoever). The remaining populations of this species also express much lower genetic diversities than found in populations of the remaining species. Noteworthy, T. fontinalis and T. tursky, even though each species has extremely small distribution range, comprises just a single population, and has 
small effective population size (1185 and 1685 respectively; based on the maximum likelihood estimates, see Table 3), express high levels of genetic diversities (Table 2).

In species comprised out of more than a single population (T. croaticus, T. karsticus and T. ukliva), a high level of isolation of populations and only very restricted gene flow has been noticed. Inside T. croaticus gene flow from Obsenica to Jadova $(\mathrm{M}=3650, \mathrm{Nm}=29)$, as well as from Suvaja to Ričica $(\mathrm{M}=1563, \mathrm{Nm}=13)$ has been estimated. The Sušik population of T. karsticus seems to be receiving immigrants from Studenac and Jasenak populations (for both population pairs $\mathrm{M}=980, \mathrm{Nm}=11$ ). Very small, bi-directional migrations have been noticed between Cetina and Vinelić populations of T. ukliva ( $\mathrm{Nm}=5$ in both directions). It is interesting that migrations were noticed among populations that are not connected with ground watercourses. Nevertheless, all estimated migration events are very small and cannot be considered as contributing significantly to size or diversity of any of the populations. For T. croaticus, particularly high levels of nucleotide diversity (0.01074 vs. 0.00506 which is the maximum value observed in the remaining species) and the total number of nucleotide differences (12.225 vs. max. 5.758 in the remaining species) were observed. Among $T$. croaticus populations those parameters were the highest in the Obsenica population, much higher than in any of the remaining populations and species.

Effective population sizes varied greatly among populations (Table 3); however, for the majority of populations they seem appropriately high regarding carrying capacities of geographically restricted karstic habitats. Expectedly, the lowest effective population size was estimated for T. karsticus population from the Studenac stream and estimates for the remaining T. karsticus populations are lower than for populations belonging to the remaining species. Besides for T. karsticus populations, low effective population size was observed also inside the Obsenica population of $T$. croaticus, whereas the remaining $T$. croaticus populations have higher effective population sizes, particularly the population from the Ričica River.

Genetic differentiation tests revealed that populations of T. karsticus and T. ukliva are genetically uniform, whereas there is genetic distinctiveness between populations of $T$. croaticus (Table 4). Namely, original hypothesis that there is no genetic distinctiveness between populations was rejected only in the case of $T$. croaticus, where permutation test revealed estimates obtained by all genetic differentiation tests to be statistically significant $(\mathrm{p}<0.05)$.

Interesting and important results were yielded by PVA analyses conducted on the endemic Telestes species and based on altogether six different scenarios (Scenario 0 encompassing the current situation, without additional threats, and Scenarios 1-5 modelling viability of the investigated Telestes populations and species in cases of possible future threats and catastrophes) (Figures 2-4). Viability of all populations and species is predicted under current conditions (probability of extinction in the next 100 years being 0). Moreover, Scenario 0 anticipates stability of the majority of populations. Exceptions are T. karsticus populations from the Studenac stream and Jasenak field for which reduction in effective population sizes is anticipated. Consequently, it is likely that effective population size of $T$. karsticus as a species will be reduced in the next 100 years, if current conditions remain. All four scenarios that comprise threats on habitats of the investigating species occurring and/or being more severe and intense (Scenarios 1-4) yielded strong prediction of all population extinction within the next 100 years. Particularly problematic seem to be Scenarios 2 and 4 , that predict very fast extinction of all endemic Telestes species (within the next 15 years). Scenario 5, encompassing catastrophic events of strong negative effects on populations, is not likely to provoke their extinction, although fluctuations and reducing of effective population sizes will probably occur under such conditions.

\section{Discussion}

Small, isolated populations inhabiting restricted areas are often considered to lose genetic diversity due to genetic drift (Frankham, 1996; Frankham, 1998; Munguia-Vega et al., 2007; Whitlock, 2000). Many other problems described by conservation genetics are also thought to be characteristic for small populations, such as Allee effect, accumulation of deleterious mutations due to mutational meltdown, decrease in genetic diversity due to selection (so called Bulmer effect), inbreeding depression etc. (de Rochambeau et al., 
2000; Whitlock, 2000). There are evidences that in very small populations, natural selection is ineffective contrary to significant genetic drift (Munguia-Vega et al., 2007) and the importance of random genetic drift is increased, acting towards loss of beneficial alleles (Whitlock, 2000). Small populations are, thereafter, usually considered particularly endangered and their future survival questionable. However, results of this investigation on the endemic Telestes species clearly show that those species are not endangered per se, just because they have small populations and are distributed in restricted areas, nor do they express genetic problems usually reported for small populations.

Almost all investigated Telestes species, despite of their extremely restricted distribution ranges and mostly small effective population sizes express moderate to high genetic diversities. During a long-term evolutionary history in their unique environments (Buj et al., 2017), they have developed adequate adaptations and also accumulated high amount of the genetic diversity. Out of the investigated species, only T. karsticus has lower genetic diversity. From conservational perspective, particularly worrying is the situation in the Studenac stream, where absolutely no genetic diversity has been recorded and all samples possess the same haplotype. Since all the remaining investigated populations express much higher levels of genetic polymorphism, situation observed in the Studenac stream, but to somewhat lesser extent also found in the Jasenak field population, are probable consequences of the local unfavorable conditions resulting in a bottleneck effect in the evolutionary history of the mentioned T. karsticus populations. Even though such results are not in accordance with the pattern more widely observed in this investigation (high genetic diversity present in small populations), this knowledge is very important from conservational perspective. Namely, without this knowledge, we might consider T. karsticus less endangered, because of its larger area of occupancy and extent of occurrence, as well as inclusion of more than a single population, than some other endemic Telestes species, for example T. polylepis, T. fontinalis and T. ukliva. However, regardless its somewhat larger distribution area and higher effective size, it has reduced evolutionary potential (because of the reduced genetic diversity) and its viability is lower than of the remaining investigated species, corroborated also by PVA analyses. Moreover, since gene flow among T. karsticus populations is almost absent, each population depends solely on its own viability and effective size, which underlies necessity for immediate, adequate conservation of those populations. Based on the obtained results, conservation plan for T. karsticusshould certainly include population augmentation, in order to prevent additional reduction in population sizes that are predicted to occur under current conditions.

On the other hand, genetic diversities observed in the remaining populations reveal that small genetic polymorphisms are not necessary character of small and isolated populations. Interestingly, contrary to the opinion that natural selection is ineffective in small size populations due to rapid genetic drift, some theoretical models (Nevo et al., 1997) suggest moderate or strong balancing selection on small populations opposing random drift and maintaining polymorphism during thousands of generations. Moreover, it was recently proposed that small populations do not continuously decline in fitness due to the fixation of slightlydeleterious mutations, but only until drift-selection balance is reached and the fixation of beneficial mutations counteracts the fixation of slightly-deleterious mutations (Poon \& Otto, 2000; Whitlock et al., 2000). For the lack of small-effect deleterious mutations in small populations, their adaptation to drift-robust fitness peaks has been proposed as an explanation (LaBar \& Adami, 2017). Namely, results of LaBar \& Adami (2017) suggested small populations to evolve to alternative areas of the fitness landscape by maintaining small-effect beneficial mutations. Although the authors propose theory of drift robustness in small populations might be true for bacterial endosymbionts and RNA viruses, their hypothesis is worth of investigation in higher organisms that naturally live in small populations, in harsh conditions of karstic watersheds and, based on our results, comprise much higher genetic diversities than expected in the presence of the significant genetic drift. Furthermore, there are literature data on other mechanisms opposing reduction of genetic diversity and loss of fitness in small populations. Lande (1994) suggested that reverse mutations (acting to return deleterious mutant alleles to the fit original forms) can substantially slow the loss of fitness. Several interesting reports (reviewed in Whitlock, 2000) pointed to possibility that the rate of beneficial mutations may even increase as mean fitness of a small population drops.

Schultz \& Lynch (1997) and Whitlock (2000) have proposed an effective population size of few hundred 
individuals to be a critical borderline below which it is likely that population will decline in fitness, but above which beneficial mutations allow the population to persist. Populations above a critical threshold size seem to be able to persist as a result of the balancing effects of fixation of beneficial alleles (Whitlock, 2000). Effective population sizes estimated for Telestes populations are mostly in the range from few hundreds to 2000 (exceptions are both T. ukliva populations and T. croaticus in the Ričica River, that have even higher effective population sizes, but also inhabit two largest rivers among the investigated water bodies, that are, moreover, permanent). Thereafter, effective population sizes of Telestes species do not seem to be too small to enable population viability based on conclusions of Whitlock (2000). For most populations it is not likely that carrying capacities in their small, karstic environments are much higher. Exceptionally high nucleotide diversity and the number of nucleotide differences noticed in $T$. croaticus, particularly in the Obsenica River corroborates pronounced genetic structuring of this species, as already revealed in previous investigation (Buj et al., 2017). Both lineages are present in the Obsenica River and their taxonomic status should further be investigated.

Based on the obtained results, investigated populations mostly seem to be isolated and gene flow estimates indicate a very low or non-existing gene flow among populations. Nevertheless, estimation of gene flow between populations inhabiting rivers that currently have no surface connections, together with their existence in streams with extremes fluctuation of the surface water level, indicates the possibility that Telestes species use underground water systems as possible shelters during dry seasons and/or as migration routes between localities that have no above ground connections. For example, gene flow between T. croaticus populations from the Obsenica and Jadova Rivers has been noticed and those two rivers have no surface connections. Similarly, two populations of T. karsticus (from Studenac and Jasenak localities) have no surface connections, but a small gene flow between them has been estimated. On the other hand, Suvaja and Ričica (inhabited by two T. croaticus populations that also exchange migrants) and Cetina and Vinalic (inhabited by two populations of T. ukliva) do have ground connections. A conclusion for migrations through underground water passages has already been reported for the genus Delminichthys, which also inhabits karstic water systems in the same area (Palandačić et al., 2012). Thereafter, possibility that fishes of the genus Telestes have similar adaptations deserves further investigation.

In addition to the important conclusion that small, endemic populations are not doomed to extinction and seem to express significant evolutionary potential and viability under current conditions, PVA results clearly pinpointed the most dangerous threats for the investigated species. Since Scenarios 2 and 4 predicted fast extinction of all investigated populations and species, their presumptions seem to be the most problematic for the endemic Telestes species. Scenario 2 encompasses negative effects of invasive species, whereas Scenario 4 predicts simultaneous effects of different threats. Expectedly, simultaneous occurrence of several threats on the endemic Telestes species would result in their joint effects reducing population sizes and provoking species extinctions extremely fast. Unfortunately, this scenario is not unlikely and cases of extinction vortex, when different environmental and genetic problems act at the same time, enlarging negative effects on the populations present one of the biggest threats for biodiversity conservation. On the other hand, Scenario 2 reveals that invasive species can be pinpointed as the most dangerous individual threat for the endemic Telestes species. Field observations corroborate this conclusion, particularly disappearance of $T$. karsticus from localities where trout species were introduced (personal observation). Invasive species are already present in habitats of several endemic Telestes species (Ćaleta et al., 2019). In some of the areas, the number of recorded non-native species even over dominates the native ones (Vukić et al., 2019). Effective removal of already present non-native species, as well as design and implementation of strategy for continuous monitoring, early discovery and quick response in order to prevent non-native species to form stabile populations in watersheds inhabited by nativeTelestes species, should be a top priority in Croatian practical nature conservation. Results of the Scenario 3 evince that, although skewed sex ratios have been recorded in various Telestes species (Marčić, 2013; Krivokapić, 2002; Zanella, 2003; Zanella et al., 2009), if this phenomenon would occur more often due to climate changes (since water temperature is likely a factor influencing sex ratio at birth), they would also result in extinction of all populations within the next 100 years. Noteworthy, in the Scenario 3, we have incalculated constant change in sex ratio and no mitigation 
effects that might occur as an adaptation of the investigated populations to climate changes. Thereafter, it is likely that effects of water temperature increment will not be as harsh as predicted by the Scenario 4 . As a conservational measure to prevent negative effects of skewed sex ratio on population viability, we can propose monitoring of sex ratio in Telestespopulations and augmentation with females from ex situ breeding programs, in cases when sex ratio would be significantly in favor of males during multiple consecutive years. Interestingly, Scenario 5, comprising very intense effects of catastrophic events occurring twice during 100 years, did not result in extinction of any of the endemic Telestes species. Since karstic environments are unfavorable and with pronounced fluctuations, it is probable that adaptations for such environments are more likely to enable survival after catastrophic events, if they do not occur too often. Thereafter, our results clearly evince that small, endemic species are by no means doomed to extinction, not do they necessarily comprise small levels of genetic diversities. On the contrary, during their evolution to specific environments, they seem to have accumulated beneficial mutations that make their fitness high and viability pronounced and high genetic diversity is surely a reservoir for coping with environmental changes and ensuring future evolutionary course of the endemic Telestes species. Conservational measures should be primarily focused on control and prevention of invasive species spreading, since they turned out to be the most dangerous threat for these unique biodiversity components.

\section{Literature}

Beerli P (1998): Estimation of migration rates and population sizes in geographically structured populations. In Carvalho, G. (ed.), Advances in Molecular Ecology., NATOASI Workshop Series IOS Press, Amsterdam: $39-53$.

Beerli P (2009): How to use Migrate or why are markov chain monte carlo programs difficult to use? In Bertorelle, G., M.W. Bruford, H. C. Hauffe, A. Rizolli \& C. Vernese (eds), Population Genetics for Animal Conservation, Vol. 17., Conservation Biology Cambridge University Press, Cambridge.

Beerli P, Felsenstein J (2001): Maximum likelihood estimation of a migration matrix and effective population sizes in $\mathrm{n}$ subpopulations by using a coalescent approach. Proceedings of the National Academy of Sciences of the USA 98: 4563-4568.

Buj I, Ćaleta M, Marčić Z, Šanda R, Vukić J, Mrakovčić M. (2015): Different histories, different destinies-impact of evolutionary history and population genetic structure on extinction risk of the Adriatic spined loaches (genus Cobitis; Cypriniformes, Actinopterygii). PLoS ONE 10(7): e0131580. doi:10.1371/journal.pone.0131580

Buj I, Marčić Z, Ćaleta M, Šanda R, Giger MF, Freyhof J, Machordom A, Vukić J (2017): Ancient connections among the European rivers and watersheds revealed from the evolutionary history of the genus Telestes (Actinopterygii; Cypriniformes). PLoS ONE 12(12): e0187366. doi:10.1371/journal.pone.018736

Buj I, Marčić Z, Čavlović K, Ćaleta M, Tutman P, Zanella D, Duplić A, Raguž L, Ivić L, Horvatić S, Mustafić P (2020): Multilocus phylogenetic analysis helps to untangle the taxonomic puzzle of chubs (genusSqualius ; Cypriniformes, Actinopteri) in the Adriatic basin of Croatia and Bosnia and Herzegovina. Zoological Journal of the Linnean Society, in press. doi:10.1093/zoolinnean/zlz133

Ćaleta M, Buj I, Mrakovčić M, Mustafić P, Zanella D, Marčić Z, Duplić A, Mihinjač T, Katavić I (2015): Croatian endemic fishes. State agency for environment protection, Zagreb.

Ćaleta M, Marčić Z, Buj I, Zanella D, Mustafić P, Duplić A, Horvatić S (2019): A review of extant Croatian freshwater fish and lampreys - Annotated list and distribution. Croatian Journal of Fisheries 77: 137-234. doi: $10.2478 /$ cjf-2019-0016.

de Rochambeau H, Fournet-Hanocq F, Vu Tien Khang J (2000): Measuring and managing genetic variability in small populations. Annales de zootechnie 49: 77-93.

Frankham R (1995): Conservation genetics. Annual Review of Genetics 29: 305-327. doi:10.1146/annurev.ge.29.120195.001513. 
Frankham R (1996): Relationships of genetic variation to population size in wildlife. Conservation Biology 10: $1500-1508$.

Frankham R (1998): Inbreding and extinction: island populations. Conservation Biology 12: 665-675.

Furlan E, Stoklosa J, Griffiths J, Gust N, Ellis R, Huggins RM, Weeks AR (2012): Small population size and extremely low levels of genetic diversity in island populations of the platypus, Ornithorhynchus anatinus . Ecology and Evolution 2(4): 844-857. doi:10.1002/ece3.195

Krivokapić M (1992): Population structure of the endemic Leuciscus souffia montenegrinus (Vuković, 1963) from the Morača River (Monte Negro, Yugoslavia). Journal of the State Nature Protection Department of the Natural Museum Podgorica 25: 83-91.

LaBar T, Adami C (2017): Evolution of drift robustness in small populations. Nature Communications 8: 1012. doi: 10.1038/s41467-017-01003-7

Lacey RC, Pollak JP (2017): VORTEX: A Stohastic Simulation of the Extinction Process. Version 10.2.6 Chicago Zoological Society, Brookfield, Illinois, USA.

Lande R (1994): Risk of population extinction from fixation of new deleterious mutations. Evolution 48: 1460-1469.

Librado P, Rozas J (2009): DnaSP v5: A software for comprehensive analysis of DNA polymorphism data. Bioinformatics 25: 1451-1452.

Marčić Z (2013): Taxonomic, biological and ecological characteristics of the genus Telestes Bonaparte, 1837 (Actinopterygii) in the area of the Velika Kapela and Mala Kapela mountains. PhD Thesis, University of Zagreb. [in Croatian].

Markert JA, Champlin DM, Gutjahr-Gobell R, et al. (2010): Population genetic diversity and fitness in multiple environments. BMC Evolutionary Biology 10: 205. doi:10.1186/1471-2148-10-205

Munguia-Vega A, Esquer-Garrigos Y, Rojas-Bracho L, Vazquez-Juarez R, Castro-Prietos A, Flores-Ramirez S (2007): Genetic drift vs. natural selection in a long-term small isolated population: major histocompatibility complex class II variation in the Gulf of California endemic porpoise (Phocoena sinus ). Molecular Ecology 16: 4051-4065. doi:10.1111/j.1365-294X.2007.03319.x

Nevo E, Kirzher V, Beiles A, Karol A (1997): Selection versus random drift: long-term polymorphism persistence in small populations (evidence and modelling). Philosophical Transactions of the Royal Society London B 352: 381-389. doi:10.1098/rstb.1997.0028

Palandačić A, Matschiner M, Zupančić P, Snoj A (2012): Fish migrate underground: the example of Delminichthys adspersus (Cyprinidae). Molecular Ecology 21: 1658-1671. doi:10.1111/j.1365294X.2012.05507.x

Poon A, Otto SP (2000): Compensating for our load of mutations: freezing the meltdown of small populations. Evolution 54: 1467-1479. doi:10.1111/j.0014-3820.2000.tb00693.x

Schultz ST, Lynch M (1997): Mutation and extinction: the role of variable mutational effects, synergistic epistasis, beneficial mutations, and degree of outcrossing. Evolution 51: 1363-1371. doi:10.1111/j.15585646.1997.tb01459.x

Vukić J, Eliášová K, Marić D, Šanda R (2019): Occurrence of alien spirlin (Alburnoides sp.) in the Neretva river basin. Knowledge and Management of Aquatic Ecosystems 420: 15. doi: 10.1051/kmae/2019007

Vuković N (1985): Ecological and biosystematics characters ofLeucisus souffia Risso, 1826 from the upper part of the Drina River, PhD Thesis, University of Sarajevo [in Bosnian].

Whitlock MC (2000): Fixation of new alleles and the extinction of small populations: drift load, beneficial alleles, and sexual selection. Evolution 54: 1855-1861. doi:10.1111/j.0014-3820.2000.tb01232.x 
Zanella D (2003): Biological and morphological characters ofLeuciscus souffia muticellus (Bonaparte, 1873) (Pisces, Cyprinidae), PhD Thesis, University of Zagreb [in Croatian].

Zanella D, Mihaljević Z, Mrakovčić M, Ćaleta M (2009): Ecology and diet of the endemic Telestes ukliva (Cyprinidae) in the Cetina River system, Croatia. Cybium 33: 97-105. doi:10.26028/cybium/2009-332-006

Table 1. Number of samples obtained from each locality and for each species, as well as accession numbers of the haplotypes deposited in the GenBank (will be added in the proof, however, alignment can be sent to Editor if needed).

\begin{tabular}{lllll}
\hline SPECIES & LOCALITY & WATERSHED & NUMBER OF SAMPLES & GENBANK ACCESSION NUMBERS \\
\hline T. croaticus & Jadova & Adriatic & 15 & \\
& Ričica & Adriatic & 4 \\
& Suvaja & Adriatic & 18 & \\
T. fontinalis & Obsenica & Adriatic & 13 & \\
T. tursky & Čikola & Black Sea & 20 & \\
T. ukliva & Cetina & Adriatic & 20 & \\
& Vinalić & Adriatic & 9 & \\
T. karsticus & Studenac & Black Sea & 5 & \\
& Sušik & Black Sea & 15 & \\
& Jasenak field & Black Sea & 6 & \\
T. polylepis & Šmitovo lake & Black Sea & 5 & \\
\hline
\end{tabular}

Table 2. Measures of intrapopulational genetic polymorphism estimated for populations of three endemic Telestes species. The remaining three species (T. polylepis, T. fontinalis, T. turskyi ) comprise a single population, so intraspecific genetic diversity is the same as intrapopulational. $\mathrm{N}$ - number of sequences, $\mathrm{S}$ - number of polymorphic sites, $\eta$ - total number of mutations, $\mathrm{H}$ - number of haplotypes, Hd -haplotype diversity, Hd_var - variance of haplotype diversity, $\mathrm{k}$ - total number of nucleotide differences, $\pi$ - nucleotide diversity.

\begin{tabular}{llllllllll}
\hline SPECIES & LOCALITY & $\mathrm{N}$ & $\mathrm{h}$ & $\eta$ & $\mathrm{S}$ & $\mathrm{Hd}$ & $\mathrm{Hd}$ _var & $\mathrm{K}$ & $\pi$ \\
\hline T. croaticus & Jadova & 15 & 8 & 29 & 29 & 0.829 & 0.00722 & 6.952 & 0.00610 \\
& Ričica & 4 & 4 & 7 & 7 & 1 & 0.03125 & 3.5 & 0.00307 \\
& Suvaja & 18 & 12 & 20 & 20 & 0.922 & 0.00260 & 5.248 & 0.00461 \\
& Obsenica & 13 & 3 & 24 & 24 & 0.513 & 0.02062 & 10 & 0.00877 \\
& TOTAL & 50 & 22 & 44 & 44 & 0.887 & 0.00116 & 12.225 & 0.01074 \\
T. fontinalis & Krbavsko field & 20 & 7 & 15 & 15 & 0.832 & 0.00237 & 2.658 & 0.00233 \\
T. turskyi & Cikola & 20 & 11 & 18 & 17 & 0.895 & 0.00271 & 4.495 & 0.00395 \\
T. ukliva & Cetina & 9 & 9 & 18 & 18 & 1 & 0.00274 & 5.389 & 0.00473 \\
& Vinalić & 5 & 5 & 14 & 14 & 1 & 0.016 & 6.6 & 0.00579 \\
T. karsticus & TOTAL & 14 & 13 & 27 & 26 & 0.989 & 0.00099 & 5.758 & 0.00506 \\
& Studenac & 10 & 1 & 0 & 0 & 0 & 0 & 0 & 0 \\
& Sušik & 15 & 7 & 9 & 9 & 0.657 & 0.01916 & 1.429 & 0.00125 \\
& Jasenak field & 6 & 2 & 1 & 1 & 0.333 & 0.0463 & 0.333 & 0.00029 \\
T. polylepis & TOTAL & 31 & 7 & 9 & 9 & 0.404 & 0.01222 & 0.757 & 0.00066 \\
& Šmit lake & 5 & 4 & 4 & 4 & 0.9 & 0.02592 & 2 & 0.00175 \\
\hline
\end{tabular}

Table 3. Effective population sizes (maximum likelihood estimates, as well as estimates at different likelihood 
percentiles) of the endemic Telestes species.

\begin{tabular}{lllll}
\hline SPECIES & POPULATION & MAXIMUM LIKELIHOOD & LIKELIHOOD AT PERCENTILES 25-75 \% & LIKELIHOC \\
\hline T. croaticus & Jadova & 1350 & $800-1900$ & $0-3265$ \\
& Ričica & 9450 & $6700-16435$ & $0-27835$ \\
& Suvaja & 2015 & $1365-2735$ & $435-4700$ \\
& Obsenica & 485 & $135-765$ & $0-1400$ \\
T. fontinalis & Krbavsko field & 1185 & $700-1600$ & $0-2400$ \\
T. tursky & Čikola & 1685 & $1135-2300$ & $265-3635$ \\
T. ukliva & Cetina & 9150 & $4500-11735$ & $2900-39265$ \\
& Vinalić & 4755 & $2482-18846$ & $1882-39091$ \\
T. karsticus & Studenac & 385 & $65-665$ & $0-1335$ \\
& Sušik & 985 & $400-1565$ & $0-3435$ \\
& Jasenak field & 485 & $0-1535$ & $*$ \\
T. polylepis & Šmit lake & 1550 & $609-2482$ & $0-8064$ \\
\hline
\end{tabular}

Table 4. Results of genetic differentiation tests among populations of species that comprise more than a single populations. $\chi^{2}$ test and HST are statistics based on haplotypes; KST, KST* ${ }^{*} \mathrm{KS}^{*}, \mathrm{Z}$ and $\mathrm{Z}^{*}$ are based on nucleotide sequences. The null hypothesis that there is no genetic diversity between two populations was rejected based on the permutation test, if $\mathrm{p}<0.05$. * indicates statistically significant statistic, ns indicates not significant.

\begin{tabular}{llllllll}
\hline Species & Statistic & Statistic & Statistic & Statistic & Statistic & Statistic & Statistic \\
\hline & $\mathrm{X}^{2}$ & Hst & Kst & Kst* & $\mathrm{Z}$ & $\mathrm{Z}^{*}$ & Snn \\
T. karsticus & $8.224(\mathrm{~ns})$ & $0.02289(\mathrm{~ns})$ & $0.00162(\mathrm{~ns})$ & $-0.01572(\mathrm{~ns})$ & $231.86(\mathrm{~ns})$ & $5.32(\mathrm{~ns})$ & $0.325(\mathrm{~ns})$ \\
T. croaticus & $94.826\left(^{*}\right)$ & $0.10949\left(^{*}\right)$ & $0.43927\left(^{*}\right)$ & $0.26778\left({ }^{*}\right)$ & $418.42(*)$ & $5.71(*)$ & $0.49433\left(^{*}\right)$ \\
T. ukliva & $14(\mathrm{~ns})$ & $0.00855(\mathrm{~ns})$ & $0.01384(\mathrm{~ns})$ & $0.02178(\mathrm{~ns})$ & $43.5(\mathrm{~ns})$ & $3.47(\mathrm{~ns})$ & $0.81(\mathrm{~s})$ \\
\hline
\end{tabular}

\section{FIGURE CAPTIONS:}

Figure 1. A map of the investigated area, with marked distribution ranges of the investigated Telestes species and sampling localities: 1-Jasenak field, 2-Studenac, 3-Sušik, 4-Šmit Lake, 5-Krbavsko field, 6-Jadova, 7Suvaja, 8-Ričica, 9-Obsenica, 10-Vinalić, 11-Cetina, 12-Čikola. Distribution ranges of different species are marked with distinct colors.

Figure 2. Results of population viability analyses for T. croaticus based on six scenarios.

Figure 3. Results of population viability analyses for T. fontinalis, T. turskyi and T. polylepis based on six scenarios.

Figure 4 Results of population viability analyses for T. uklivabased on six scenarios.

Figure 5. Results of population viability analyses for T. karsticus based on six scenarios.

Data Accessibility Statement: All newly obtained DNA haplotypes are deposited in the GenBank under the following accession numbers: WILL BE ADDED IN TE PROOF.

Competing Interests Statement: Authors declare no competing interests.

Authors Contribution Section:

Ivana Buj : conceptualization (lead), formal analyses, investigation, original draft preparation, supervision. Zoran Marčić : conceptualization, investigation, review \& editing, visualization. Elena Flauder : formal 
analyses, investigation.Marko Ćaleta : investigation, review \& editing. Radek Šanda : investigation, formal analyses, review \& editing. Jasna Vukić : conceptualization, investigation, review \& editing.

Acknowledgements: IB received support from the SYNTHESYS Programme project CZ-TAF-4228 at the National Museum Prague, financed by European Community Research Infrastructure Action under the FP7 "Capacities" Programme. JV was supported by the institutional resources of the Ministry of Education, Youth, and Sports of the Czech Republic. RS by the Ministry of Culture of the Czech Republic (DKRVO 2019-2023/6.IV.b National Museum, 00023272).
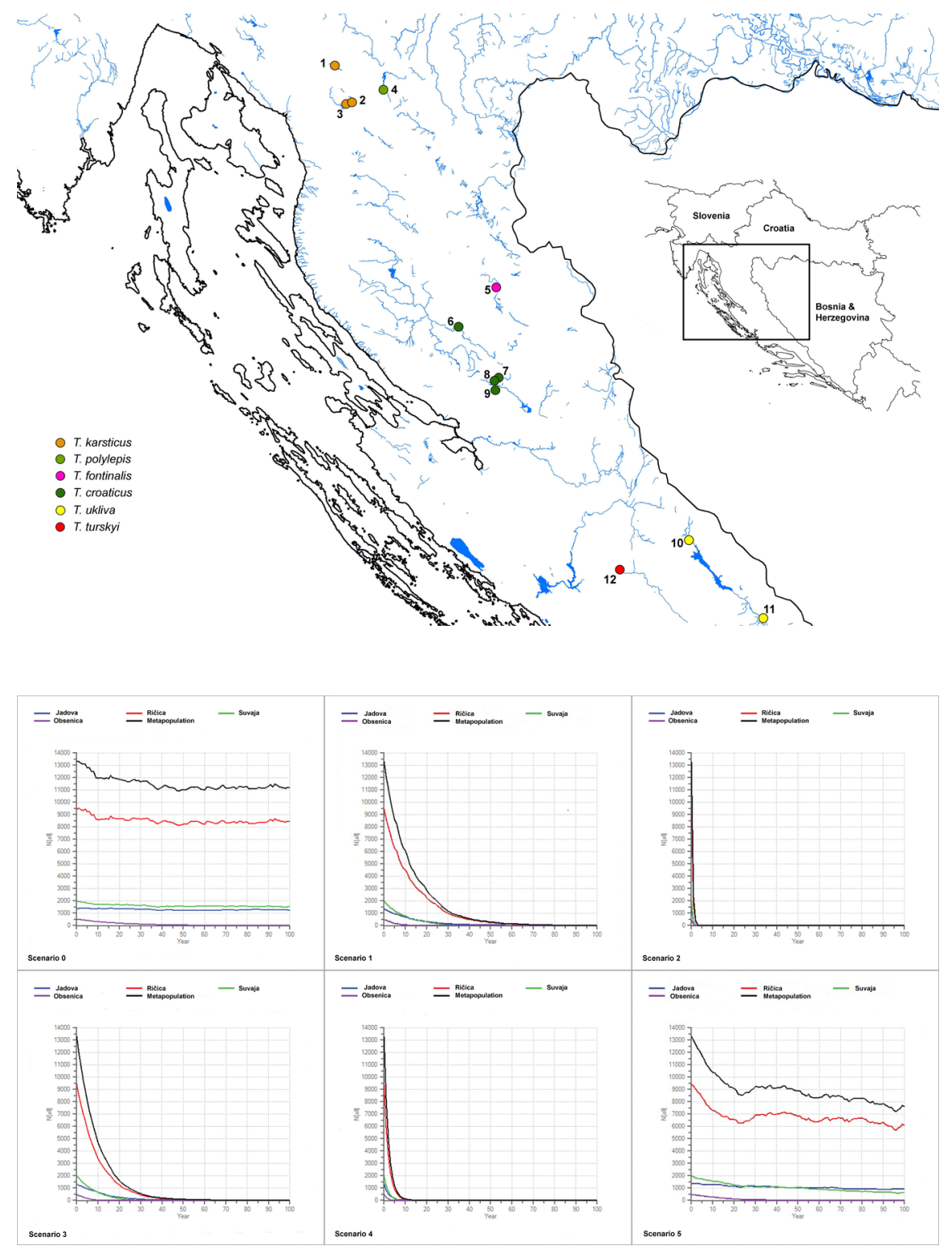

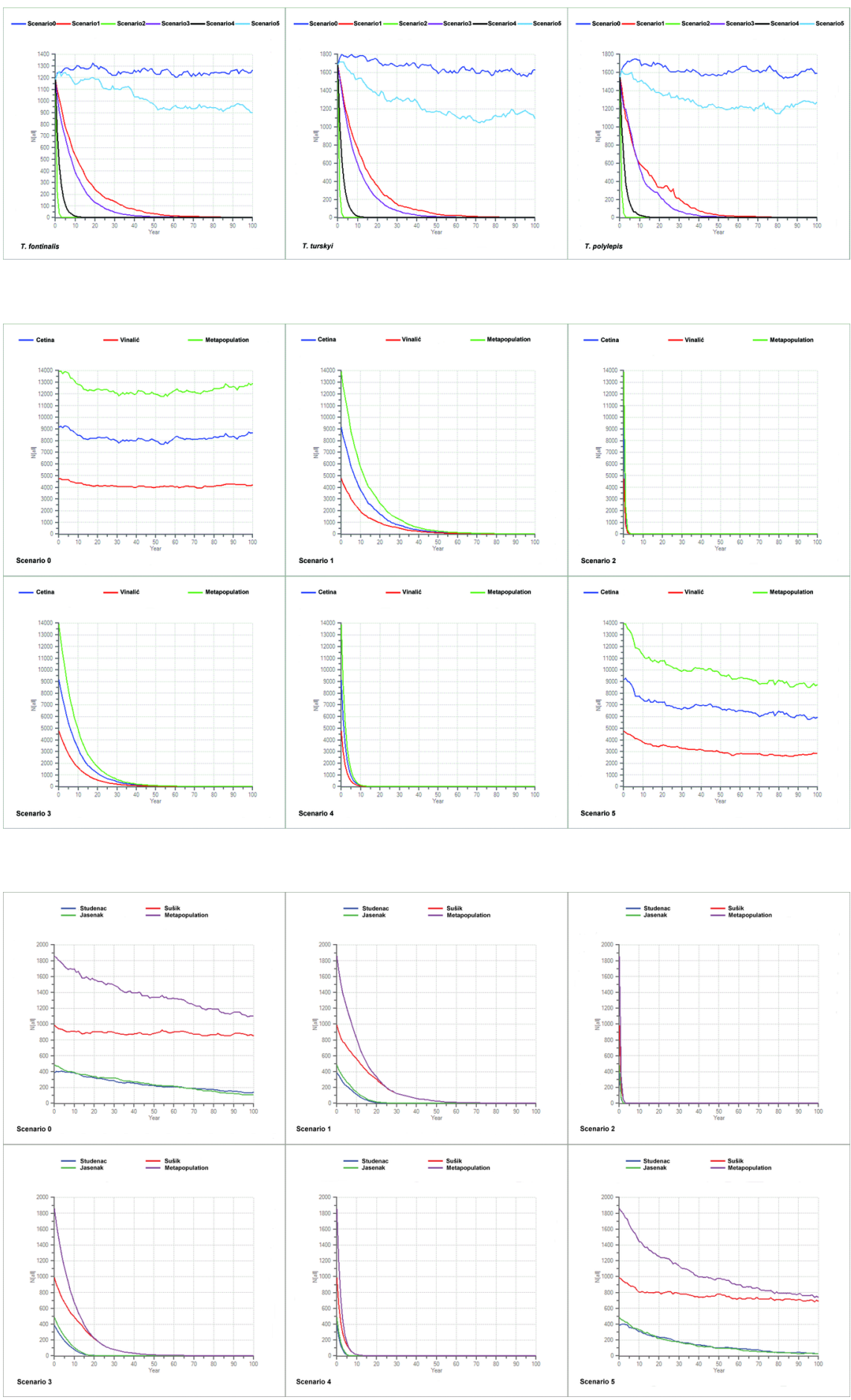